\title{
Prolonged seizure like activity after sevoflurane based anesthesia
}

\begin{abstract}
Sevoflurane is one of the most popular and widely use inhalational anesthetics in modern day anaesthesia as it has many advantages over the previously used inhalational agents. Although it has many favorable properties, very little is known about its property of causing seizure like activities associated with its use. We encountered a case of sevoflurane related prolonged seizure like activities (myoclonic jerky movement with alter consciousness) in the postoperative period requiring antiepiletic medications and postoperative intensive care admission.
\end{abstract}

Keywords: inhalational agents, sevoflurane, seizures, anesthesia
Volume 6 Issue 2 - 2016

\author{
Naveen Aggarwal,' Monica Gupta,' Surjya \\ Prasad Upadhyay ${ }^{2}$ \\ 'Specialist Anaesthesiologist, Al salam International Hospital, \\ Kuwait \\ ${ }^{2}$ Specialist Anaesthesiologist, NMC Hospital, UAE
}

Correspondence: Surjya Prasad Upadhyay, Specialist

Anaesthesiologist, NMC hospital, DIP Dubai, UAE,

Email surjya.upadhyay@nmc.ae

Received: October 26, 2016 | Published: November 25, 2016

\section{Introduction}

Sevoflurane is well known and accepted by all of the anesthesiologists for its use in both children and adult population due to its distinct advantages over other conventional volatile agents ${ }^{1}$ such as, rapid onset and emergence from anesthesia because of low solubility in blood, non-irritant and of low pungency, can be safely used in both children and adult for induction and maintenance. Although Sevoflurane is well tolerated in majority of patients, occasional adverse effects have been reported like coughing, laryngospasm, tachycardia, hypertension, emergence delirium and agitation. ${ }^{2}$ Very transient self limiting seizure like activities has been reported with sevoflurane anaesthesia. ${ }^{3}$ We encountered prolonged seizure like activities in a 5 years old child in the postoperative period following Sevoflurane based anaesthesia for tonsillectomy, necessitating use of antiepileptic mediation to control the seizure and postoperative intensive care monitoring.

\section{Case report}

A 5 year old child weighting $23 \mathrm{~kg}$ with American Society of Anesthesiology (ASA) physical status 1 was scheduled for elective Coblation assisted tonsillectomy (CAT). There was a history of use nebulization with salbutamol and beclomethasone for 2-3 days as outpatient during a flu attack 2 months ago. He had no other systemic illness. There was no history of epilepsy, or febrile seizure, or hospitalization in the past. General and physical examination was unremarkable and there was no sign and symptoms of any respiratory tract infections or hyper reactivity.

Informed written consent was taken from parents and surgery was scheduled at 09:00hrs. The child was kept fasted for solid and milk after midnight except for water and apple juice which was allowed until 07:00hrs. Before shifting to operative room, child was given buccal midazolam $7 \mathrm{mg}$ orally 30 minutes before the scheduled surgery. When the child was calm and cooperative, shifted to OT table and basic monitors (pulse oximetry, noninvasive blood pressure, ECG) were attached. As there was no intravenous canula in place, the child was induced by inhalational anaesthetic with increasing concentration of sevoflurane ( $8 \%$ within $2 \mathrm{~min}$ ) with $50 \%$ oxygen in nitrous oxide, intravenous (IV) access was established once the child was adequately anaesthetised. IV fentanyl $25 \mathrm{mcg}$ and cis-atracurium $4 \mathrm{mg}$ was used to facilitate tracheal intubation, which was accomplished smoothly under direct laryngoscopy with no 5 cuff preform portex Ring Adair Elwin (RAE) orotracheal tube which was fixed at $16 \mathrm{~cm}$ mark at lip level after confirming its correct position by bilateral auscultation and waveform capnography. Sevoflurane concentration reduced to $2 \%$ after tracheal intubation. Child was maintained on controlled ventilation with Tidal volume of $180 \mathrm{ml}$ with respiratory rate of 20 , on $50 \%$ nitrous with oxygen. IV dexamethasone $2 \mathrm{mg}$ was given before the surgical incision, to reduce the nausea vomiting and also prolong the analgesia. Child was stable throughout the surgical procedure which lasted for about 35 minutes. Intraoperatively, the child received IV paracetamol $300 \mathrm{mg}$ and $25 \mathrm{mg}$ of diclofenac suppository rectally for postoperative analgesia. Ondanestron $2 \mathrm{mg}$ was given as prophylaxis for nausea and vomiting. At the end of the surgery, sevoflurane and nitrous oxide were discontinued, neuromuscular blocking was reversed with neostigmine $1 \mathrm{mg}$ along with glycopyrrolate $0.2 \mathrm{mg}$; trachea was extubated when the child was fully conscious and regained adequate muscle strength. The child was shifted to post anaesthesia care unit (PACU) in left lateral decubitus position with bed side monitors and oxygen by facemask at 4 lit per minute.

After about 10 minutes in PACU, child was found to have abnormal jerky, seizure like movements involving the whole body. These abnormal movements were occurring in repeated bursts of short duration of 15-20 seconds. Between these jerky movement, child was drowsy, but arousable to deep pain, but hemodynamic and respiration was maintained. Immediately, IV midazolam $2 \mathrm{mg}$ was given to controlled the jerky movement. Following IV midazolam, the myoclonic movement still continued for about $10 \mathrm{~min}$, but with decrease in frequency and intensity. The child was maintaining and protecting the airway throughout these episodes. Haemodynamics was stable throughout these events. During these events, blood sample were sent for electrolytes, blood sugar and ABG (arterial blood gas); which were all within the normal limit. Neurologist opinion was sought as we could not find any cause for such seizure like activity. There were no neurological deficits and neurologist made a provisional diagnosis of myoclonic seizures which may be induced by high concentration of sevoflurane given intraoperatively. The child was shifted to pediatric ICU for observation and monitoring. There were few myoclonic twitches even after 2 hours for which the child was put on $400 \mathrm{mg}$ sodium valproate orally and all abnormal movements were subsided gradually over next 6 hours. No abnormality was detected on Computed Tomography (CT) brain and electroencephalograph (EEG). 
The child was kept in ICU overnight and was discharged home after 2 days uneventfully. Sodium valproate was gradually tapper off within 7 days by the neurologist. The child was in regular follow up until 3 months postoperatively, so far has not been reported any myoclonic activity.

\section{Discussion}

Sevoflurane is a methyl ether volatile anesthetic agent commonly used for induction and maintenance of anesthesia in both pediatric and adult patients because of its various favorable properties. Although it is well tolerated by majority of patients, there are reports of serious adverse effects such as coughing, laryngospasm, desatuaration, sympathetic stimulation causing tachycardia and hypertension and emerge delirium and agitation associated with use of high concentration of sevoflurane. Sevoflurane has been reported to be associated with transient seizure like electrical activity or myoclonic movements that are often self limiting. ${ }^{3-6}$ Although the agitation associated with sevoflurane anesthesia is not linked to seizure as evidence by absence of typical epileptiform activity in EEG $;^{7}$ however, its use is controversial in neurosurgery or in patients with epilepsy. ${ }^{8}$

Sevolfurane and myoclonic seizure like activities is a controversial issues. There are very few literatures and case reports available that suggests a correlation between use of sevoflurane and myoclonic or seizure like activities. ${ }^{3,9,10}$ Sevoflurane has been reported to cause more epileptiform activities than isoflurane in patients with known epilepsy, but when sevoflurane is combined with nitrous oxide, there is no increase in such activities. ${ }^{10}$ Sato et al. ${ }^{11}$ using electrocorticograph in a series of patients with the normal brain, found abnormal burst and spike activities associated with high end tidal sevoflurane concentration (more than 3\%). Similarly, Vakkuri et al. ${ }^{12}$ used high dose of sevoflurane induction (8\%) with nitrous oxide observed high incidence of several abnormal EEG activities during induction in paediatric patients. On the other hand, Constant and coworkers did not find any seizures in children during inhalational induction with sevoflurane. ${ }^{7}$

In recent studies, it has been seen that inhalational induction with high concentration of sevoflurane is associated with tachycardia and hypertension; such sympathetic stimulation is common during concurrent epileptiform discharges seen with enflurane anaesthesia. So, the hypersympathetic stimulation may be an autonomic manifestation of underlying epileptic activities in the brain. ${ }^{13-15}$ In our case, there was no significant changes in vitals parameters neither intraoperatively nor postoperatively, even during the seizure like activities.

There are numerous factors responsible for seizures or abnormal myoclonic activities in perioperative period. Patients with history of seizure disorder, perioperative hypoxia, hypocarbia, hypoglycemia, electrolyte disturbance or inadequate anesthesia and analgesia and epileptiform drug are the main causative factors for seizure like activities in postoperative period. None of the factor was present in our patient; we attributed the cause of abnormal seizure like activities in our patient to high concentration of sevoflurane anesthesia. There is no consensus guideline or recommendation of the safe concentration of sevoflurane to avoid such rare adverse effect, care must be paid during induction and maintenance with high concentration of sevoflurane especially patients prone to have epileptic activities. Combining with other intravenous and or inhalational agent, greater use of regional anaesthesia techniques can reduce the requirement sevolurane and thereby, possibly reduces the occurrence of such rare adverse effect.

\section{Conclusion}

Although rare, high concentration of sevoflurane can induce abnormal seizure like myoclonic activities in both healthy patients and in patients with history of epilepsy. There is no consensus guideline or recommendation of the safe concentration of sevoflurane. Multimodal strategies like combine use of intravenous and inhalational anesthetics, regional anaesthesia can be used to limit the concentration of sevoflurane and occurrence of such adverse effect.

\section{Acknowledgments}

None.

\section{Conflicts of interest}

The authors declare there are no conflicts of interest.

\section{Funding}

None.

\section{References}

1. O'Keeffe N. Volatile Anesthetics. In: Healy TEJ, Knight Pr, editors. Wylie and Churchill-davidson's A practice of Anesthesia. London: Arnold; 2003:523-542.

2. Goa KL, Noble S, Spencer CM. Sevoflurane in paediatric anaesthesia. Paediatr Drugs. 1999;1(2):127-153.

3. Terasako K, Ishii S. Postoperative seizure -like activity following sevoflurane anesthesia. Acta Anaesthesiol Scand. 1996;40(8 Pt 1):953954

4. Adachi M, Ikemoto Y, Kubo K, et al. Seizure like movements during induction of anesthesia with sevoflurane. Br J Anaesth. 1992;68(2):214 215

5. Woodforth IJ, Hicks RG, Crawford MR, et al. Electroencephalographic evidence of seizure under deep sevoflurane anaesthesia in a nonepileptic patient. Anesthesiology. 1997;87(6):1579-1582.

6. Komatsu H, Taie S, Endo S, et al. Electrical seizures during sevoflurane anesthesia in two pediatric patients with epilepsy. Anesthesiology. 1994;81(6):1535-1537.

7. Constant I, Dubois MC, Piat V, et al. Changes in electroencephalogram and autonomic cardiovascular activity during induction of anesthesia with sevoflurane compared with halothane in children. Anesthesiology. 1999;91(6):1604-1615.

8. Jaas kelainen SK, Kaisti K, Sunil L, et al. Sevoflurane is epileptogenic in health subjects at surgical levels of anesthesia. Neurology. 2003;61(8):1073-1078.

9. Akeson J, Didriksson I. Convulsions on anesthetic induction with sevoflurane in young children. Acta Anaesthesiol Scand. 2004;48(4):405-407.

10. Iljima T, Nakamura Z, Iwao Y, et al. The epileptogenic properties of the volatile anesthetics sevoflurane and isoflurane in patients with epilepsy. Anesth Analg. 2000;91(4):989-995.

11. Sato K, Shamoto H, Kato M. Efeect of sevoflurane on electrocorticogram in normal brain. J Neurosurg Anesthesiol. 2002;14(1):63-65.

12. Vakkuri A, Yli Hankala A, Sarkela M, et al. Sevoflurane mask induction of anesthesia is associated with epileptiform EEG in children. Acta Anaesthesiol Scand. 2001;45:805-811.

13. Jantti V, Yli-Hankala A. Correlation of instantaneous heart rate and EEG suppression during enflurane anesthesia: synchronous inhibition of heart rate and cortical electrical activity. Electroencephalogr Clin Neurophysiol. 1990;76(5):476-479.

14. Constant I, Seeman R, Murat I. Sevoflurane and epileptiform EEG changes. Paediatr Anaesth. 2005;15(4):266-274. 